\title{
Statistical analysis of the determinations of the Sun's Galactocentric distance
}

\author{
Zinovy Malkin ${ }^{1,2}$ \\ ${ }^{1}$ Pulkovo Observatory, St. Petersburg 196140, Russia \\ ${ }^{2}$ St. Petersburg State University, St. Petersburg 198504, Russia \\ email: malkin@gao.spb.ru
}

\begin{abstract}
Based on several tens of $\mathrm{R}_{0}$ measurements made during the past two decades, several studies have been performed to derive the best estimate of $R_{0}$. Some used just simple averaging to derive a result, whereas others provided comprehensive analyses of possible errors in published results. In either case, detailed statistical analyses of data used were not performed. However, a computation of the best estimates of the Galactic rotation constants is not only an astronomical but also a metrological task. Here we perform an analysis of $53 \mathrm{R}_{0}$ measurements (published in the past 20 years) to assess the consistency of the data. Our analysis shows that they are internally consistent. It is also shown that any trend in the $\mathrm{R}_{0}$ estimates from the last 20 years is statistically negligible, which renders the presence of a bandwagon effect doubtful. On the other hand, the formal errors in the published $\mathrm{R}_{0}$ estimates improve significantly with time.
\end{abstract}

Keywords. Galaxy: center, Galaxy: fundamental parameters

\section{Introduction}

Accurate knowledge of the distance from the Sun to the center of the Galaxy, $\mathrm{R}_{0}$, is important in many fields of astronomy and space science. In particular, the primary motivation for this study was a wish to improve the accuracy of modeling the Galactic aberration (Malkin 2011).

Over the past decades, many tens of $\mathrm{R}_{0}$ determinations have been made, making use of different principles and observing methods, and thus characterized by different random and systematic errors. Therefore, deriving a best $R_{0}$ estimate from these data is not only an astronomical but also a metrological task, similar to deriving the best estimates of the fundamental constants in physics. For the latter, several statistical methods have been developed to obtain best estimates - as well as their realistic uncertainties - from heterogeneous measurements. In this study, we applied those methods to the $\mathrm{R}_{0}$ data.

Another goal of this study was to investigate a possible trend in the multi-year series of $\mathrm{R}_{0}$ estimates, as discussed by many authors. However, estimates of any such trend differ significantly among papers. Therefore, we tried to clarify this issue using the latest results. More details are provided by Malkin (2012).

\section{Data used}

For the present study, we used all $\mathrm{R}_{0}$ measurements published in the period 1992 2011 , with the exception of a number of results that were revised in subsequent papers. We did not use the results of Glushkova et al. (1998; revised by Glushkova et al. 1999), Paczynski \& Stanek (1998; revised by Stanek et al. 2000), Eisenhauer et al. (2003; revised by Eisenhauer et al. 2005, which was in turn revised by Gillessen et al. 2009). In total, 53 estimates (listed in Table 1) were used. 
Table 1. $\mathrm{R}_{0}$ estimates used in this study.

\begin{tabular}{|c|c|c|}
\hline $\mathrm{R}_{0}(\mathrm{kpc})$ & $\pm(1 \sigma)$ & Reference \\
\hline 7.9 & 0.8 & Merrifield, M.R. 1992, AJ, 103, 1552 \\
\hline 8.1 & 1.1 & Gwinn, C.R., et al. 1992, ApJ, 393, 149 \\
\hline 7.6 & 0.6 & Moran, J.M., et al. 1993, Lect. Notes Phys., 412, 244 \\
\hline 7.6 & 0.4 & Maciel, W.J. 1993, Ap\&SSS, 206, 285 \\
\hline 8.09 & 0.3 & Pont, F., et al. $1994, A \& S A, 285,415$ \\
\hline 7.5 & 1.0 & Nikiforov, I.I., \& Petrovskaya, I.V. 1994, Astron. Rep., 38, 642 \\
\hline 7.0 & 0.5 & Rastorguev, A.S., et al. 1994, Astron. Lett., 20, 591 \\
\hline 8.8 & 0.5 & Glass, I.S., et al. 1995, MNRAS, 273,383 \\
\hline 7.1 & 0.5 & Dambis, A.K., et al. 1995, Astron. Lett., 21, 291 \\
\hline 8.3 & 1.0 & Carney, B.W., et al. $1995, A J, 110,1674$ \\
\hline 8.21 & 0.98 & Huterer, D., et al. 1995, AJ, 110, 2705 \\
\hline 7.95 & 0.4 & Layden, A.C., et al. 1996, AJ, 112, 2110 \\
\hline 7.55 & 0.7 & Honma, M., \& Sofue, Y. 1996, PASJ, 48, L103 \\
\hline 8.1 & 0.4 & Feast, M.W. 1997, MNRAS, 284, 761 \\
\hline 8.5 & 0.5 & Feast, M., \& Whitelock, P. 1997, MNRAS, 291, 683 \\
\hline 7.66 & 0.54 & Metzger, M.R., et al. 1998, AJ, 115, 635 \\
\hline 8.1 & 0.15 & Udalski, A. 1998, Acta Astron., 48, 113 \\
\hline 7.1 & 0.4 & Olling, R.P., \& Merrifield, M.R. 1998, MNRAS, 297, 943 \\
\hline 8.51 & 0.29 & Feast, M., et al. $1998, M N R A S, 298, \mathrm{~L} 43$ \\
\hline 8.2 & 0.21 & Stanek, K.Z., \& Garnavich, P.M. 1998, ApJ, 503, L131 \\
\hline 8.6 & 1.0 & Surdin, V.G. 1999, Astron. Astrophys. Trans., 18, 367 \\
\hline 7.4 & 0.3 & Glushkova, E.V., et al. 1999, Astron. Astrophys. Trans., 18, 349 \\
\hline 7.9 & 0.3 & McNamara, D.H. et al. 2000, PASP, 112, 202 \\
\hline 8.67 & 0.4 & Stanek, K.Z., et al. 2000, Acta Astron., 50, 191 \\
\hline 8.2 & 0.7 & Nikiforov, I.I. 2000, Astron. Soc. Pac. Conf. Ser., 209, 403 \\
\hline 8.24 & 0.42 & Alves, D.R. $2000, A p J, 539,732$ \\
\hline 8.05 & 0.6 & Genzel, R., et al. $2000, M N R A S, 317,348$ \\
\hline 8.3 & 0.3 & Gerasimenko, T.P. 2004, Astron. Rep., 48, 103 \\
\hline 7.7 & 0.15 & Babusiaux, C., \& Gilmore, G. 2005, MNRAS, 358, 1309 \\
\hline 8.01 & 0.44 & Avedisova, V.S. 2005, Astron. Rep., 49, 435 \\
\hline 8.7 & 0.6 & Groenewegen, M.A.T., \& Blommaert, J.A.D.L. 2005, $A \& A, 443,143$ \\
\hline 7.2 & 0.3 & Bica, E., et al. $2006, A \& A A, 450,105$ \\
\hline 7.52 & 0.36 & Nishiyama, S., et al. 2006, ApJ, 647, 1093 \\
\hline 8.1 & 0.7 & Shen, M., Zhu, Z. 2007, Chin. J. Astron. Astrophys., 7, 120 \\
\hline 7.4 & 0.3 & Bobylev, V.V., et al. 2007, Astron. Lett., 33, 720 \\
\hline 7.94 & 0.45 & Groenewegen, M.A.T., et al. $2008, A \varepsilon \mathcal{E} A, 481,441$ \\
\hline 8.07 & 0.35 & Trippe, S., et al. $2008, A \mathscr{E} A, 492,419$ \\
\hline 8.16 & 0.5 & Ghez, A.M., et al. 2008, ApJ, 689, 1044 \\
\hline 8.33 & 0.35 & Gillessen, S., et al. 2009, ApJ, 692, 1075 \\
\hline 8.7 & 0.5 & Vanhollebeke, E., 2009, $A \mathscr{E} A, 498,95$ \\
\hline 7.58 & 0.40 & Dambis, A.K. $2009, M N R A S, 396,553$ \\
\hline 7.2 & 0.3 & Bonatto, C., et al. 2009, Globular Clusters: Guides to Galaxies, p. 209 \\
\hline 8.4 & 0.6 & Reid, M.J., et al. 2009, ApJ, 700, 137 \\
\hline 7.75 & 0.5 & Majaess, D.J., et al. $2009, M N R A S, 398,263$ \\
\hline 7.9 & 0.75 & Reid, M.J., et al. 2009, ApJ, 705,1548 \\
\hline 8.24 & 0.43 & Matsunaga, N., et al. $2009, M N R A S, 399,1709$ \\
\hline 8.28 & 0.33 & Gillessen, S., 2009, ApJ, 707, L114 \\
\hline 7.7 & 0.4 & Dambis, A.K. 2010, Variable Stars, the Galactic halo and Galaxy Formation, p. 177 \\
\hline 8.1 & 0.6 & Majaess, D. 2010, Acta Astron., 60, 55 \\
\hline 8.3 & 1.1 & Sato, M., et al. $2010, A p J, 720,1055$ \\
\hline 7.80 & 0.26 & Ando, K., et al. $2011, P A S J, 63,45$ \\
\hline 8.3 & 0.23 & Brunthaler, A., et al. 2011, Astron. Nachr., 332, 461 \\
\hline 8.29 & 0.16 & McMillan, P.J. 2011, MNRAS, 414, 2446 \\
\hline
\end{tabular}

Where both random (statistical) and systematic uncertainties were given, they were summed in quadrature. If two different values were given for the lower and upper boundaries of the confidence interval, the mean value of these boundaries was used as the uncertainty in the result (the lower and upper boundaries were close to each other in all cases, so that this approximation procedure does not significantly affect the final result). Where authors gave several estimates of $R_{0}$ without a final preference, the unweighted average of these estimates was computed. 


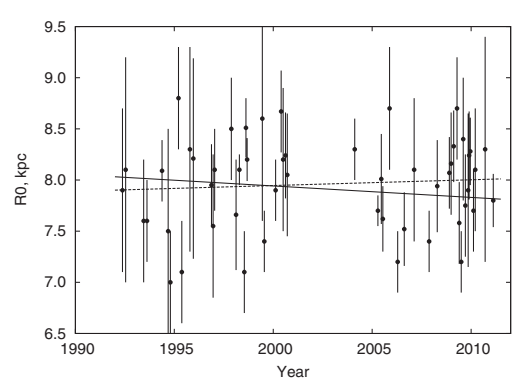

Figure 1. $\mathrm{R}_{0}$ estimates used in this study. The weighted (solid line) and unweighted (dashed line) trends are also shown.

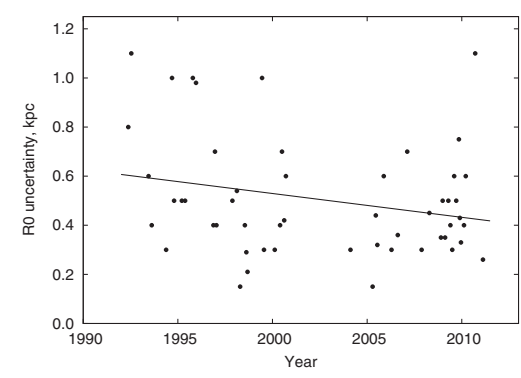

Figure 2. Uncertainty in $\mathrm{R}_{0}$ estimates used in this study.

\section{Results}

Our analysis used two approaches. First, we investigated a possible drift in the $\mathrm{R}_{0}$ values (see Fig. 1), which may indicate the presence of a bandwagon effect. We obtained

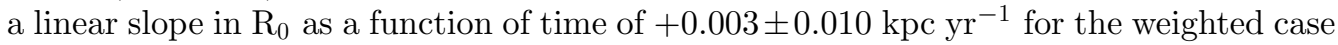
and $+0.009 \pm 0.010 \mathrm{kpc} \mathrm{yr}^{-1}$ for the unweighted case. Thus, any trend in $\mathrm{R}_{0}$ estimates published in the last 20 years is statistically insignificant, which renders a significant bandwagon effect doubtful. This conclusion is confirmed by application of the Abbe criterion (Malkin 2013). The latter paper also contains a detailed discussion on this subject.

On the other hand, the trend in the reported $\mathrm{R}_{0}$ uncertainties (see Fig. 2) is statistically

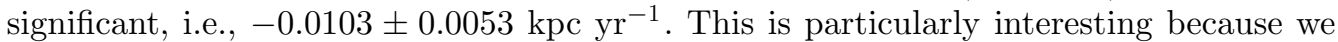
expect that there are two tendencies in the changes in the errors in $R_{0}$ measurements with time. First, the errors should decrease with progress in observational and analysis methods. On the other hand, one can expect that the published errors should increase because most authors of recent papers now pay more attention to the correct computation of the uncertainties in their result. Evidently, the former tendency prevails.

To assess the internal consistency of the $\mathrm{R}_{0}$ measurements, several statistical methods were used. They can be divided into two groups. The first group consists of computation of the unweighted and classical weighted means, the author's modification with respect to computation of the mean uncertainty (Malkin 2001), and three other weighted-mean modifications, which allow to account for input data discrepancies, i.e., limitation of relative weights (Nichols 2004), normalized residuals (James et al. 1992), and the MandelPaule method (Paule \& Mandel 1982). If all variants of the weighted mean produce the same mean value and standard error, the input data are consistent. The second group of methods includes determination of the median with error estimation (Müller 1995) and bootstrap median methods. Detailed descriptions of these methods are given in Malkin (2012). The results of the computations are presented in Table 2.

\section{Concluding remarks}

Although the published $\mathrm{R}_{0}$ estimates were obtained based on different methods and samples of objects, they are consistent rather than discrepant. The results of the computation of a mean $\mathrm{R}_{0}$ value obtained using different statistical techniques converge at the level of approximately $0.1 \mathrm{kpc}$, which is much smaller than the uncertainty in the average value. Note, however, that this conclusion is not evident. Similar analysis of $\Omega_{0}$ measurements has shown that the latter are much less consistent. 
Table 2. Average estimates of $\mathrm{R}_{0}$ obtained with different statistical techniques (see text).

\begin{tabular}{lc}
\hline \multicolumn{1}{c}{ Method } & $R_{0}, \mathrm{kpc}$ \\
\hline Unweighted mean & $7.979 \pm 0.061$ \\
Classical weighted mean & $7.967 \pm 0.048$ \\
Modified weighted mean & $7.967 \pm 0.073$ \\
Limitation of relative weights & $7.967 \pm 0.048$ \\
Normalized residuals & $7.967 \pm 0.048$ \\
Mandel-Paule method & $7.958 \pm 0.058$ \\
Median & $8.090 \pm 0.062$ \\
Bootstrap median & $8.060 \pm 0.072$ \\
\hline
\end{tabular}

As significant experience in deriving best estimates of the physical constants has shown, using various statistical methods to evaluate the best $R_{0}$ estimate is very important to assess data consistency and obtain a realistic uncertainty in the final result. Therefore, careful astronomical consideration of the published measurements should be accompanied by a careful statistical analysis. It should be recognized that the computation of the new conventional IAU $\mathrm{R}_{0}$ value is not only an astronomical, but also a metrological task.

Another result of this study is that any trend in the $\mathrm{R}_{0}$ estimates obtained during the last 20 years is statistically insignificant, which makes it unlikely that $\mathrm{R}_{0}$ results are significantly affected by a bandwagon effect. On the other hand, the formal errors in the published $\mathrm{R}_{0}$ estimates improve significantly with time.

Note that the average value $\mathrm{R}_{0}=8.0 \pm 0.25 \mathrm{kpc}$ computed in this study differs from the results of the latest direct measurements obtained from stellar orbits about Sgr $\mathrm{A}^{*}$, trigonometric parallaxes to Sgr B2, and over 60 trigonometric parallaxes of masers, which give $\mathrm{R}_{0}=8.4 \pm 0.2 \mathrm{kpc}$ (M. J. Reid, priv. comm.), although these values are still statistically consistent.

On the other hand, it seems important to properly combine all results obtained based on different methods, because this provides better systematic stability of the average result. Indeed, the systematic and random errors of all these results should be assessed and the corresponding correction should be applied when possible before averaging (although this is a very difficult task).

\section{References}

Eisenhauer, F., Schödel, R., Genzel, R., et al. 2003, ApJ, 597, L121

Eisenhauer, F., Genzel, R., Alexander, T., et al. 2005, ApJ, 628, 246

Glushkova, E. V., Dambis, A. K., Mel'nik, A. M., \& Rastorguev, A. S. 1998, A\&A, 329, 514

James, M. F., Mills, R. W., \& Weaver, D. R. 1992, Nucl. Instr. Meth. Phys. Res. A, 313, 277

Malkin, Z. 2001, Comm. Inst. Appl. Astron., 137

Malkin, Z. M. 2011, Astron. Rep., 55, 810

Malkin, Z. 2012, arXiv:1202.6128

Malkin, Z. 2013, Astron. Rep., 57, 128

Müller, J. W. 1995, Report BIPM-95/2, Sèvres, France

Nichols, A. L. 2004, Appl. Rad. Isot., 60, 247

Paczyński, B. \& Stanek, K. Z. 1998, ApJ, 494, L219

Paule, R. \& Mandel, J. 1982, J. Res. Nat'l Bur. Stand., 87, 377 